\title{
Role of Mirror Therapy for Phantom Limb Pain in Below Knee Amputees
}

\author{
Dr. Poonji Gupta ${ }^{1,}$ Dr. Amit Saraf Mbbs $\mathrm{Ms}^{2}$ \\ ${ }^{1}$ Junior Resident ( MBBS )Govt medical college, Patiala \\ ${ }^{2}$ Associate Professor Dept. of Orthopaedics SIMS, Unnao, ( Lucknow ) \\ Corresponding author: * Dr. Amit Saraf Mbbs Ms
}

\begin{abstract}
:
Introduction: The pain caused by surgery is usually of a transient nature, however the perception of pain in an amputated limb often persists. This prolonged pain, which is often refractory to pain-killing medication, nerve block and surgical treatment may severely affect the patient's quality of life. The phenomenon of phantom limb pain has been investigated using neurological, neurophysiological and psychopathological approaches. However exact cause of phantom limb pain is still a mystery. In this study we analysed the role of mirror therapy for treatment of phantom limb pain in below knee amputation.

Material and methods: 96 patients who had phantom limb pain after below knee amputation were included in this study. They had to visit the hospital four times a week for a 15-minute treatment period. They performed movement of unaffected limb while watching its mirror reflection and thus creating a visual illusion of movement of affected limb. The degree of pain relief was measured on visual analog scale (VAS).

Results: 70 patients out of 96 reported an improvement of 4 or more degrees of VAS score after 6 months of the treatment. The result was statistically significant.

Conclusions: Mirror therapy improves pain sensation of the amputated part when other treatment modalities fail. This therapy works on the principle of mirror neuron system. A mirror neuron fires either when a person acts or when a person observes same action performed by another. The mirror image of the normal body part helps reorganize and integrate the mismatch between proprioception and visual feedback of the removed body. This reorganization decreases the sense or emotion of phantom limb pain in the amputated part.
\end{abstract}

Key words: Mirror image therapy, phantom limb pain, below knee amputation

\section{Introduction}

Phantom limb pain is a false pain perception in a body part which has been removed or lost. Ambrose Pare, first reported this sensation in $16^{\text {th }}$ century. Many studies on phantom limb pain have been done after that especially on patients who lost their limbs during the First and Second World Wars. These studies investigated phantom limb pain using neurological, neurophysiological and psychopathological approaches. However exact cause of phantom limb pain is still a mystery (1).

More than $2 / 3^{\text {rd }}$ of phantom limb pain develops after amputation. It can develop immediately after an amputation or may sometimes take a few days. About $75 \%$ of the patients experience the pain within a few days after amputation. This prolonged pain, which is often refractory to pain-killing medication, nerve block and surgical treatment may severely affect the patient's quality of life (2-4). In this study we analyzed the role of mirror therapy for treatment of phantom limb pain in below knee amputation.

\section{Material and methods}

This study was conducted in Christian medical College, Ludhiana from March 2014 till October 2015. 96 patients who had phantom limb pain after below knee amputation were included in this study. Patients with previous neurological symptoms, diabetic nephropathy, bilateral amputees and those who were severely crippled to visit hospital daily were excluded from this study.

A proper consent was taken from all the patients before they were included in the study.

Included patients had to visit the hospital four times a week for a 15 -minute treatment period. In this technique they were seated in front of a long mirror. They were advised to perform active movements of all the joints of unaffected while watching its mirror reflection and thus creating a visual illusion of movement of affected limb. The degree of pain relief was measured using visual analog scale (VAS) after one, three and six months of therapy. A Visual Analogue Scale (VAS) is a measurement instrument that tries to measure a characteristic or attitude that is believed to range across a continuum of values and cannot easily be directly 
measured. A horizontal line of $100 \mathrm{~mm}$ in length was drawn, anchored by word descriptors at each end, as illustrated below.

No pain Very severe pain

The patients were asked to mark on the line the point that they feel represents their perception of their current state. The VAS score is determined by measuring in millimetres from the left hand end of the line to the point that the patient marks (5).

\section{Results}

After one month of therapy, there was an improvement of 4 or more degrees of VAS score in $15 \%$ of patients. After three months, $40 \%$ patients showed improvement. At six months of treatment 70 patients out of 96 reported an improvement of 4 or more degrees of VAS score. The result was statistically significant wit $p$ value $<0.05$.

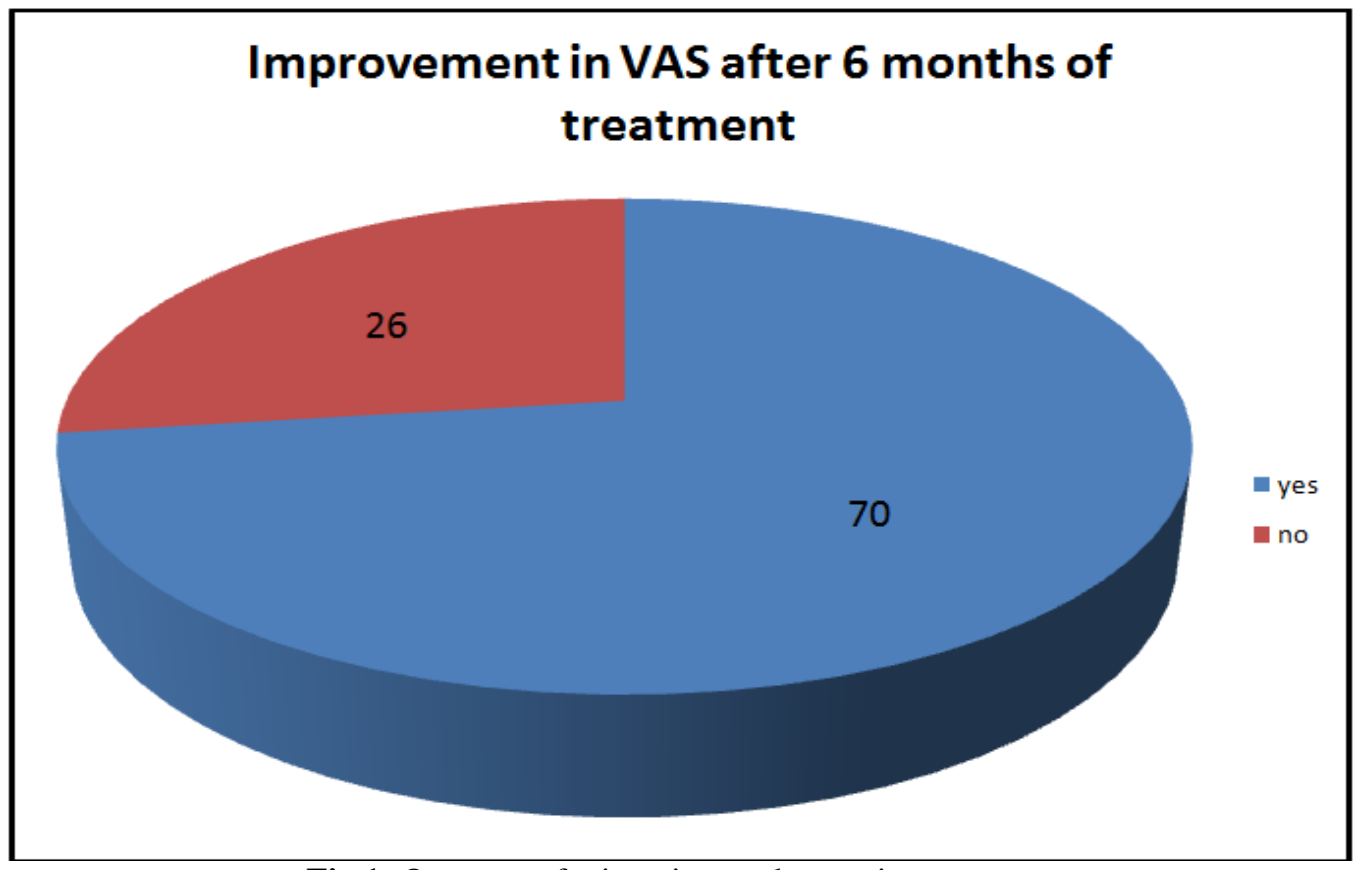

Fig 1: Outcome of mirror image therapy in amputees

\section{Discussion}

Mirror therapy was unveiled in 1996 by Ramachandran. During this therapy, a patient is allowed to feel the imaginary movement of the removed body part behaving as normal body movement while performing movements of intact limb in front of a mirror. This therapy works on the principle of mirror neuron system. A mirror neuron fires either when a person acts or when a person observes same action performed by another. The mirror image of the normal body part reorganizes and integrate the mismatch between proprioception and visual feedback of the removed body. This reorganization decreases the sense or emotion of phantom limb pain in the amputated part (6). In the present study itself a significant improvement of 4 or more degrees of VAS score after six months of therapy proves that mirror image therapy is an effective treatment to reduce phantom limb pain in below knee amputees.

\section{Bibliography}

[1]. Sae Young Kim, Yun Young Kim, Mirror Therapy for Phantom Limb Pain, Korean J Pain. 2012 Oct; 25 (4): $272-274$.

[2]. Carlen PL, Wall PD, Nadvorna H, Steinbach T. Phantom limbs and related phenomena in recent traumatic amputations. Neurology. 1978;28:211-217

[3]. Hayes C, Armstrong-Brown A, Burstal R. Perioperative intravenous ketamine infusion for the prevention of persistent postamputation pain: a randomized, controlled trial. Anaesth Intensive Care. 2004;32:330-338

[4]. Nikolajsen L, Ilkjaer S, Krøner K, Christensen JH, Jensen TS. The influence of preamputation pain on postamputation stump and phantom pain. Pain. 1997;72:393-405

[5]. Wewers M.E. \& Lowe N.K. A critical review of visual analogue scales in the measurement of clinical phenomena. Research in Nursing and Health. $1990 ; 13,227-236$

[6]. McCabe CS, Haigh RC, Halligan PW, Blake DR. Simulating sensory-motor incongruence in healthy volunteers: implications for a cortical model of pain. Rheumatology (Oxford) 2005;44:509-516. 